\title{
Curvature-induced capillary interaction of spherical particles at a liquid interface
}

\author{
Alois Würger \\ CPMOH, CNRS-Université Bordeaux 1, 351 cours de la Libération, 33405 Talence, France
}

\begin{abstract}
We consider a liquid interface with different principal curvatures $\pm c$ and find that the mere presence of a spherical particle leads to a deformation field of quadrupolar symmetry; the corresponding "capillary quadrupole moment" is given by the ratio of the particle size and the curvature radius. The resulting pair interaction of nearby particles is anisotropic and favors the formation of aggregates of cubic symmetry. Since the single-particle trapping energy depends quadratically on curvature with a negative prefactor, a curvature gradient induces a lateral force that pushes the particles towards strongly curved regions of the interface. As an illustration we discuss the effects occuring on a catenoid.
\end{abstract}

\section{INTRODUCTION}

Capillary interactions arise from the deformation of a liquid phase boundary due to the presence of colloidal particles. They influence the phase behavior of twodimensional colloids [1] and are relevant for technological applications such as nanostructuring of surfaces [2-4]. In the visible range, the balance of gravity and interface tension gives rise to a variety of phenomena, as a simple example we note the aggregation of cereals on a bowl of milk. Tension, weight, and hydrodynamics concur in the locomotion of water walking insects and in the meniscus climbing of the larvae of the waterlily leaf beetle $[5,6]$. For small particles in the micron or nanometer range, gravity is negligible and often supplanted by charge effects. It has been known for a while that two-dimensional colloidal crystals of ionized particles are stabilized by electrostatic repulsion $[1,7]$. More recently, the experimental observation of an attractive force [8] has stimulated a detailed study of the charge-induced electric stress on the interface [9-12]; indeed, like charges trapped on micron-sized water droplets are subject to an attractive capillary potential that depends on the mean curvature of the liquid interface [13].

An external force $F$ on a spherical particle floating on an interface of tension $\gamma$, induces an isotropic deformation field that varies logarithmically with the distance, $\xi=(F / 2 \pi \gamma) \ln r$ [14]. On the other hand, the mere presence of a non-spherical particle may give rise to an anisotropic interface deformation; like in bulk elasticity, the dipolar term vanishes, and in most cases the leadingorder deformation is given by the known quadrupolar field in $2 \mathrm{D}, \xi \sim \cos (2 \varphi) r^{-2}$ [15-18]; its prefactor is proportional to the capillary quadrupole moment carried by the particle. The resulting pair potential is anisotropic and favors the formation of clusters with strong orientational order. In recent years, several experimental studies have confirmed the relevance of capillary quadrupole interactions [15, 19].

In the examples mentioned so far, capillary phenomena arise from the properties of the trapped particles, such as weight, charge, or shape. In other words, a spherical massless and uncharged particle would not affect the interface. In the present paper, we discuss an exception to this rule: We show that spherical particles at an interface with two different principal curvatures interact as if they carried a capillary quadrupole; the value of the effective quadrupole moment is given by the ratio of the particle size and the curvature radius of the interface. After a general formulation of the problem we perform a perturbative expansion in terms of the small-gradient approximation and determine the minimal surface by varying the deformation field at fixed boundary conditions. The expression for the change of interface area provides the trapping potential of a single particle and the effective pair interaction. As an example, we study the lateral forces on micron sized particles on a catenoid interface, and finally discuss related problems occuring for a twophase flow in a microchannel and for the meniscus climbing of water treading insects.

\section{THE INTERFACE AREA}

Consider a particle trapped at a liquid interface. The total interface energy $E=\gamma S_{L}+\gamma_{1} P_{1}+\gamma_{2} P_{2}$ depends on the tension of the fluid phase boundary $\gamma$, those of the particle surface in the two liquids, $\gamma_{1}$ and $\gamma_{2}$, and on the corresponding areas $S_{L}, P_{1}, P_{2}$. The global constraint of minimum energy readily leads to two local constraints, one on the liquid interface and one on the intersection of the three surfaces: In the absence of external forces, the fluid phase boundary satisfies Laplace's equation, whereas the contact angle at the particule surface is given by Young's relation

$$
\cos \theta_{0}=\frac{\gamma_{1}-\gamma_{2}}{\gamma}
$$

(For $\left|\gamma_{1}-\gamma_{2}\right|>\gamma$ the particle does not stay at the interface but is soluted in one of the fluid phases.) These conditions do not depend explicitly on the solid-liquid surface areas $P_{1}$ and $P_{2}$; yet they do in an implicit manner, since the contact line, which constitutes the boundary of $P_{1}$ and $P_{2}$, is determined by Young's angle.

We briefly discuss the simple case of a spherical particle of radius $r_{0}$ at a flat liquid interface. Because of the axial symmetry of the problem, the two constraints given above are independent of each other. The flat interface 
is still the relevant solution of Laplace's equation, and Young's angle is satisfied by adjusting the vertical position of the particle. Moreover, this angle describes the position of the contact line with respect to the particle center; and the contact line is given by a circle of radius

$$
a_{0}=r_{0} \sin \theta_{0} .
$$

The presence of the particle reduces the interface area by a disk of area $\pi a_{0}^{2}$, corresponding to the trapping energy $\gamma \pi a_{0}^{2}$.

In this work we consider a more complicated situation that arises at a curved liquid interface. The trapped particles reduce the interface area and thus lower its energy. Because of the finite curvature, their presence also modifies the interface profile and thus renders the evaluation of the area significantly more difficult. From Fig. 1 it is clear that the inhomogeneous curvature tensor breaks the axial symmetry. As a consequence, the constraints at the liquid interface and the contact line are no longer independent of each other; indeed, Young's relation (1) leads to a non-uniform boundary condition for the interface profile which, in turn, results in an interface deformation.

In mathematical terms, the problem consists in minimizing the liquid interface area with the constraint on the contact angle along the two-phase line at the particle surface. The area $S_{L}$ of the fluid phase boundary with $N$ particles is given by the integral

$$
\iint_{\mathcal{I}} d u d v \sqrt{g}
$$

which is parameterized by two real variables $u$ and $v$. The determinant of the metric tensor,

$$
g=\operatorname{det} g_{i j}, \quad g_{i j}=\partial_{i} \mathbf{R} \cdot \partial_{j} \mathbf{R},
$$

is defined in terms of the derivatives of the position vector $\mathbf{R}(u, v)$. It turns out convenient to substract from $S_{L}$ the area $S_{0}$ of the unperturbed interface without particles, and to consider their difference $S=S_{L}-S_{0}$. Starting from the known parameterization $\mathbf{R}_{0}(u, v)$ of the interface in the absence of colloidal particles, and denoting the corresponding determinant $g_{0}$, the change of area reads

$$
S=\iint_{\mathcal{I}} d u d v \sqrt{g}-\iint_{\mathcal{I}+\mathcal{P}} d u d v \sqrt{g_{0}} .
$$

Here $\mathcal{P}$ and $\mathcal{I}$ denote the range of the parameters $u, v$ occupied by the particles and the liquid interface, respectively, and $\mathcal{I}+\mathcal{P}$ accounts for the interface in the absence of particles. Each particle corresponds to a hole in the interface, which is bounded by the contact line $\partial S$. Note that $S$ is always negative; for a flat interface one has $g=1=g_{0}$, and $N$ particles reduce the area by $S=-N \pi a_{0}^{2}$.

Evaluation of Eq. (2) requires us to caracterize the boundary $\partial S$ and to determine the interface deformation that satisfies the condition of mechanical equilibrium. Fig. 1 shows the intersection of a spherical particle and a surface of zero mean curvature. One easily realizes that the contact line is not a circle but a curve in 3D space, and that the unperturbed interface does not satisfy Eq. (1). Yet Young's relation expresses the condition of mechanical equilibrium that must be satisfied at the contact line and thus requires a deformation of the interface in the vicinity of the particles. This constraint is implemented through the scalar product of the normal vector of the particle surface $\mathbf{n}_{P}$ and that of the interface $\mathbf{n}_{I}$. In terms of the function

$$
F(\mathbf{r})=\mathbf{n}_{P} \cdot \mathbf{n}_{I}-\cos \theta_{0},
$$

mechanical equilibrium corresponds to $F(\mathbf{r})=0$ along the contact line. Formally, this is achieved by adding to the interface area a line integral,

$$
S+\oint_{\partial S} d s \lambda(\mathbf{r}) F(\mathbf{r})
$$

where the function $\lambda(\mathbf{r})$ plays the role of a generalized Lagrange multiplier. The interface profile is determined by minimizing $S$ and requiring that the functional derivatives with respect to $\lambda(\mathbf{r})$ vanishes.

\section{PERTURBATION THEORY}

\section{A. The area functional}

The above expression for the area takes a simple form in the Monge gauge, where $u$ and $v$ are Euclidean coordinates that define the plane $A$ tangent to the surface in a given point, and where $w(u, v)$ is the height of the surface with respect to the plane $A$. One readily finds the determinant of the metric tensor $g=1+(\nabla w)^{2}$.

Since capillary deformations induced by colloidal particles are weak in general, we resort to the small-gradient approximation

$$
|\nabla w| \ll 1 .
$$

where the $2 \mathrm{D}$ gradient operator $\nabla$ is defined with respect to the plane $A$. (This inequality is valid at distances smaller than curvature radii.) Thus the interface

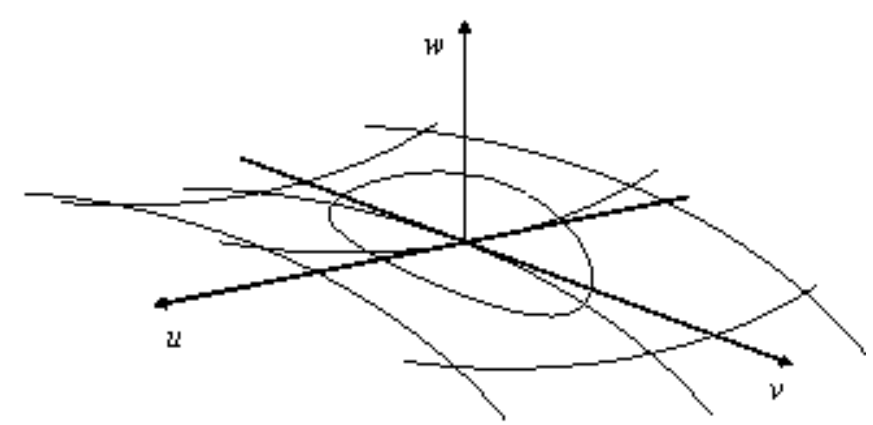

FIG. 1: Contact line of a spherical particle at a curved interface. The axes define the local coordinates $u, v, w$. 


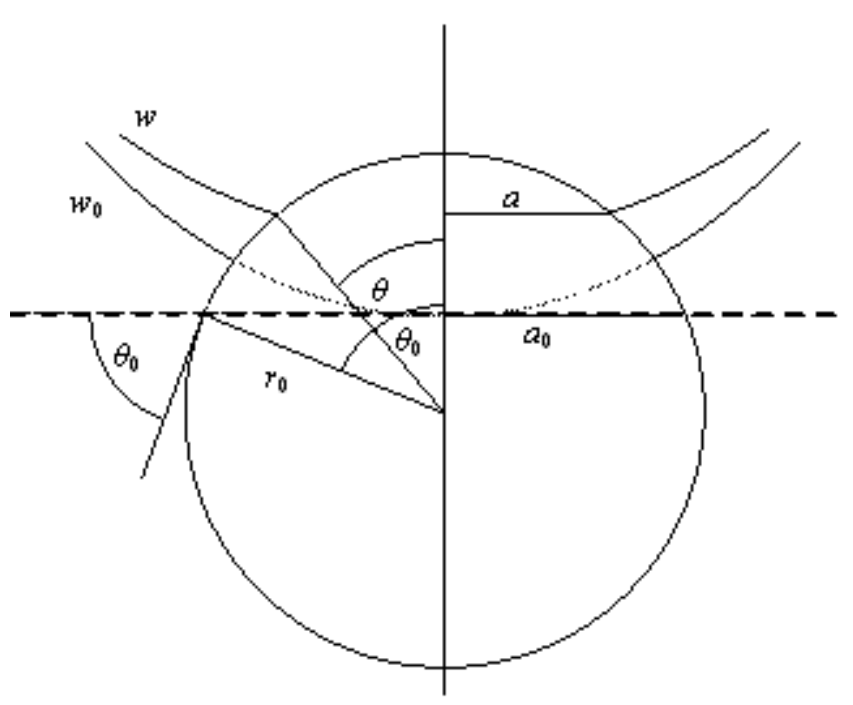

FIG. 2: Schematic view of a particle of radius $r_{0}$ at a liquid interface. $\theta_{0}$ and $a_{0}$ are the contact angle and the radius of the contact line for a flat interface. $w_{0}$ describes a curved interface without particle, $w=w_{0}+\xi$ accounts for the deformation due to the particle. $\theta$ is the polar angle of the deformed contact line, and $a$ its radial position.

area may be approximated by the first terms of a series in powers of the deformation field $w$ and its derivatives. The determinant reads to quadratic order

$$
\sqrt{g}=1+\frac{1}{2}(\nabla w)^{2}+\ldots
$$

It turns out convenient to write the deformation field as

$$
w=w_{0}+\xi
$$

where $w_{0}$ describes the unperturbed surface with the determinant

$$
\sqrt{g_{0}}=1+\frac{1}{2}\left(\nabla w_{0}\right)^{2}+\ldots
$$

and where $\xi$ accounts for the deformation due the presence of the colloidal particles.

When regrouping the integrals over the interface domain $\mathcal{I}$, the area change due to the presence of colloidal particles takes the form

$$
S=\int_{\mathcal{I}} d A\left(\sqrt{g}-\sqrt{g_{0}}\right)-\int_{\mathcal{P}} d A \sqrt{g_{0}}
$$

The second term represents the area occupied by the particle, whereas the first one accounts for the deformationinduced area difference on the domain $\mathcal{I}$. Inserting the truncated series for $g$ and $g_{0}$, the latter becomes $\frac{1}{2} \int d A\left((\nabla w)^{2}-\left(\nabla w_{0}\right)^{2}\right)$; its sign clearly depends on the ratio of the gradients of the deformed and unperturbed surfaces. Using $w=w_{0}+\xi$, we thus obtain the functional

$$
\begin{array}{r}
S[\xi]=\frac{1}{2} \int_{\mathcal{I}} d A\left((\nabla \xi)^{2}+2 \nabla \xi \cdot \nabla w_{0}\right) \\
-\int_{\mathcal{P}} d A\left(1+\frac{1}{2}\left(\nabla w_{0}\right)^{2}\right) .
\end{array}
$$

We still have to specify the contact line $\partial S$ and the constraint $F(\mathbf{r})$ in terms of the deformation field $w$, and to give the explicit form of $w_{0}$.

\section{B. The contact line}

In local coordinates, the contact line is determined by the radial variable $a$ and the height function $w$ evaluated at the particle surface, $\tilde{w}=\left.w\right|_{\partial S}$. For further use, we express $a$ as a function of $\tilde{w}$ and give its power series trunated at second order. From Fig. 2, one readily finds the cosine and the sine of the polar angle $\theta$ in terms of $\tilde{w}$ and $a$,

$$
\cos \theta=\cos \theta_{0}+\frac{\tilde{w}}{r_{0}}, \quad \sin \theta=\frac{a(\tilde{w})}{r_{0}}
$$

Rewriting $a(\tilde{w})=r_{0} \sqrt{1-\cos ^{2} \theta}$ as a function of the small parameter

$$
\tilde{w} / a_{0} \ll 1
$$

and expanding to quadratic order, one has

$$
a(\tilde{w})=a_{0}-\tilde{w} \cot \theta_{0}-\frac{1}{2} \frac{\tilde{w}^{2}}{a_{0}}\left(\cot ^{2} \theta_{0}+1\right)+\ldots
$$

The vertical and radial coordinates of the contact line, $\tilde{w}$ and $a$, are parameterized by the azimutal angle $\varphi$; a schematic view of $a(\tilde{w}(\varphi))$ is given in Fig. 3 .

\section{Boundary condition}

Eq. (3) gives the contact angle in terms of the vectors normal on the particle surface and the liquid interface. Chosing polar coordinates $r, \varphi$ in the $u-v$-plane and defining the corresponding local basis $\mathbf{e}_{r}, \mathbf{e}_{\varphi}, \mathbf{e}_{w}$, the normal vectors read

$$
\mathbf{n}_{P}=\sin \theta \mathbf{e}_{r}+\cos \theta \mathbf{e}_{w}, \quad \mathbf{n}_{I}=\left(\mathbf{e}_{w}-\nabla \tilde{w}\right) / \sqrt{g} .
$$

With the scalar product $\mathbf{n}_{P} \cdot \mathbf{n}_{I}$ and the geometric relations (9) we obtain

$$
F(\mathbf{r})=\frac{1}{r_{0 \sqrt{g}}}\left[\tilde{w}-a(\tilde{w}) \mathbf{e}_{r} \cdot \nabla \tilde{w}+(1-\sqrt{g}) r_{0} \cos \theta_{0}\right]
$$

Since both $a$ and $g$ depend on $\tilde{w}$, this expression is a non-linear function of $\tilde{w}$. The linear approximation is 


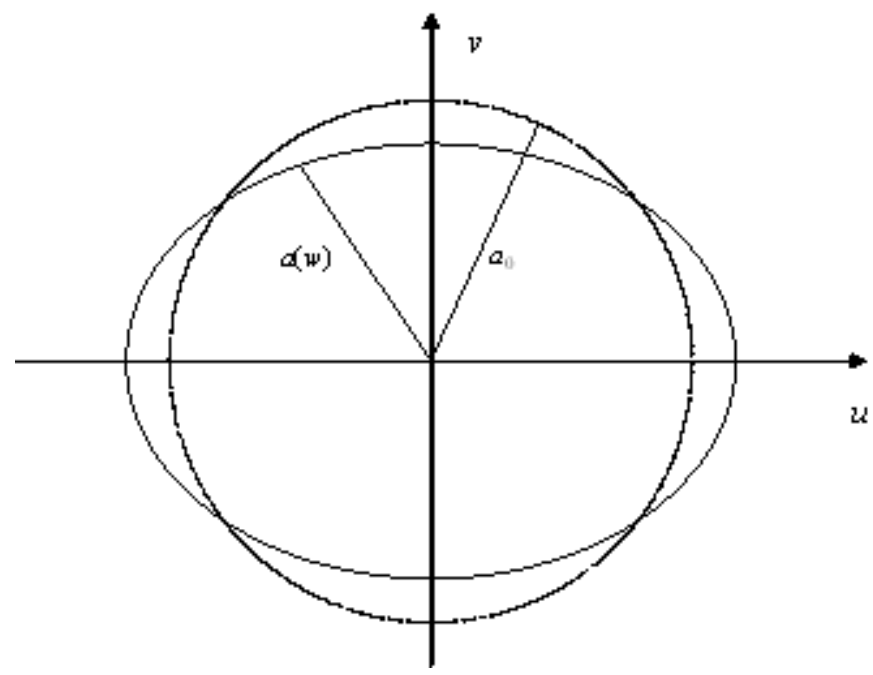

FIG. 3: The contact line of a spherical particle on a deformed surface is given by the vertical position $\tilde{w}$ and the radial coordinate $a(\tilde{w})$. On a flat interface, the contact line forms a circle of radius $a_{0}$ in the $u-v$-plane.

achieved by inserting the expansions (6) and (10), and by retaining the leading terms only,

$$
F(\mathbf{r})=\frac{\tilde{w}\left(a_{0}, \varphi\right)}{a_{0}}-\left.\mathbf{e}_{r} \cdot \nabla \tilde{w}(r, \varphi)\right|_{r=a_{0}} .
$$

Note that this relation applies to the deformation field along the contact line.

\section{The unperturbed surface}

Since the quantities $w_{0}$ and $\xi$ defined in (7) turn out to be of the same order of magnitude, it is justified to treat them on an equal footing and to expand $w_{0}$ in terms of the small gradient approximation (5). Any minimal surface has zero mean curvature; as a consequence, the equation of the undeformed surface satisfies $\nabla^{2} w_{0}=0$ and can be cast in the form

$$
w_{0}(\mathbf{r})=\frac{1}{2} c\left(u^{2}-v^{2}\right)+\ldots .
$$

where the principal curvatures at the origin are given by $\pm c$. For later use we rewrite this expression in polar coordinates, $r=\sqrt{u^{2}+v^{2}}$ and $\varphi=\arctan (v / u)$, and thus have

$$
w_{0}(\mathbf{r})=\frac{1}{2} c r^{2} \cos (2 \varphi)
$$

The condition $\left|\nabla w_{0}\right| \ll 1$ restricts the validity of the expansion for $w_{0}(\mathbf{r})$ to distances $r$ within the curvature radius, $r \ll 1 / c$. (We have supposed that the principal curvatures do not vary significantly in this range.)

\section{VARIATIONAL PRINCIPLE}

The change of area (8) comprises two terms $S=S_{\mathcal{I}}+$ $S_{\mathcal{P}}$, that involve integrals over the parameter domains $\mathcal{I}$ and $\mathcal{P}$ occupied by the liquid interface and the particles, respectively. Here we derive explicit expressions in terms of the deformation field at the contact line.

Integrating the first term in (8) by parts, we find

$$
S_{\mathcal{I}}=\oint_{\partial S} d \mathbf{s} \cdot\left(\frac{1}{2} \nabla \xi+\nabla w_{0}\right) \xi-\frac{1}{2} \int d A \xi \nabla^{2} \xi
$$

Since the integrand is already of second order in the small quantities $\xi$ and $w_{0}$, we replace the oriented line element along the surface boundary $\partial S$ by the leading term $d \mathbf{s}=$ $-\mathbf{e}_{r} a_{0} d \varphi$, and thus have

$$
S_{\mathcal{I}}=-a_{0} \int_{0}^{2 \pi} d \varphi\left(\frac{1}{2} \partial_{r} \xi+\partial_{r} w_{0}\right) \xi-\frac{1}{2} \int d A \xi \nabla^{2} \xi .
$$

The second contribution $S_{\mathcal{P}}$ represents the area occupied by the particle; it is defined with respect to the unperturbed interface and bounded by the contact line $\partial S$. At a flat interface it reads $-\pi a_{0}^{2}$; we are mainly interested in the small changes that arise from the finite curvature of $w_{0}$ and from the non-circular shape of the contact line. We evaluate $S_{\mathcal{P}}$ for a single particle located at the origin. In polar coordinates we have $d A=d \varphi d r r$ and $\left(\nabla w_{0}\right)^{2}=c^{2} r^{2}$, and the radial integral is readily performed,

$$
S_{\mathcal{P}}=-\int_{0}^{2 \pi} d \varphi\left(\frac{1}{2} a(\tilde{w})^{2}+\frac{1}{8} c^{2} a(\tilde{w})^{4}\right) .
$$

Inserting (10), expanding to quadratic order in the small parameters $c a_{0}$ and $\tilde{w} / a_{0}$, integrating the terms that involve $w_{0}$ only, and using $\int d \varphi w_{0}=0$, we find

$$
\begin{aligned}
S_{\mathcal{P}} & =-\pi a_{0}^{2}\left(1+\frac{1}{8} c^{2} a_{0}^{2}\right) \\
& +\int_{0}^{2 \pi} d \varphi\left(\xi a_{0} \cot \theta_{0}+\xi w_{0}+\frac{\xi^{2}}{2}\right) .
\end{aligned}
$$

Eqs. (13) and (14) explicit the functional dependence of the area on the interface profile $\xi$, where the actual deformation is to be determined by minimizing the surface area. Since $S_{\mathcal{P}}$ and the first term in $S_{\mathcal{I}}$ involve the deformation at the contact line and thus are fixed by the boundary condition (11), the variation with respect to $\xi$ reduces to

$$
0=\frac{\delta S}{\delta \xi}=-\nabla^{2} \xi
$$

which is the well-known condition for a minimal surface.

The boundary condition is formally imposed by the functional derivative $\delta S / \delta \lambda=0$, which implies $F(\mathbf{r})=0$ everywhere along the contact line. Inserting the form 
(12) and the relation $d w_{0} / d r=2 w_{0} / r$ in (11), we obtain the linear inhomogeneous differential equation

$$
\left.a_{0} \frac{d \xi(r, \varphi)}{d r}\right|_{r=a_{0}}=\xi\left(a_{0}, \varphi\right)-w_{0}\left(a_{0}, \varphi\right) .
$$

Together with the condition that $\xi$ vanishes at large distances, Eqs. (15) and (16) entirely determine the deformation field; their solution is denoted $\hat{\xi}(\mathbf{r})$. Since the boundary condition (16) is a linear equation and since the integral of the inhomogeneity vanishes, $\int d \varphi w_{0}=0$, a similar relation holds for its solution, and the corresponding integral in (14) vanishes, $\int d \varphi \hat{\xi} a_{0} \cot \theta_{0}=0$.

The surface integral in (13) is zero because of $\nabla^{2} \xi=0$. Replacing the derivatives in the line integral with (16) and $d w_{0} / d r=2 w_{0} / r$, the integrand simplifies to $\frac{1}{2} \hat{\xi}^{2}+$ $\frac{3}{2} \hat{\xi} w_{0}$; taking the sum $S=S_{\mathcal{I}}+S_{\mathcal{P}}$, the terms in $\hat{\xi}^{2}$ cancel, and we obtain the total change of area

$$
S_{1}[\hat{\xi}]=-\pi a_{0}^{2}\left(1+\frac{1}{8} c^{2} a_{0}^{2}\right)-\frac{1}{2} \int_{0}^{2 \pi} d \varphi \hat{\xi} w_{0} .
$$

The constant represents the area that the particle occupies on the unperturbed interface, whereas the integral term accounts for the additional reduction due to the relaxation of the interface profile.

This result has been obtained for a single particle at the origin. The formal generalization is straightforward,

$$
S_{N}=-N \pi a_{0}^{2}\left(1+\frac{1}{8} c^{2} a_{0}^{2}\right)-\frac{1}{2} \sum_{i=1}^{N} \int_{0}^{2 \pi} d \varphi_{i} \hat{\xi}_{i} w_{0},
$$

with a set of local coordinates for each of the $N$ particles. Here $\hat{\xi}_{i}$ represents the total deformation field evaluated at the contact line of particle $i$; since it comprises contributions from all particles, care has to be taken when evaluating the boundary condition (16). In the remainder of this paper, we determine the deformation field $\hat{\xi}$ for the particular cases $N=1,2$, and we derive both the trapping energy of a single particle and the pair interaction of two nearby particles.

\section{SINGLE-PARTICLE CASE}

We consider the deformation field induced by a single particle. The solution of the Young-Laplace equation $\nabla^{2} \xi=0$ reads in polar coordinates $r, \varphi$

$$
\xi(\mathbf{r})=\xi_{0} \ln r+\sum_{n=1}^{\infty} \xi_{n}\left(\frac{a_{0}}{r}\right)^{n} \cos \left(n \varphi-\delta_{n}\right),
$$

where the coefficients $\xi_{m}$ and the phases $\delta_{m}$ are to be determined from the boundary condition and the requirement that the deformation vanishes at large distances.

Inserting the above series for $\xi$ and the explicit form of the inhomogeneity (12) in the differential equation (16), one readily finds that $n=2$ is the only finite term. The solution reads

$$
\hat{\xi}(\mathbf{r})=\beta_{1} f(\mathbf{r})
$$

with the shape function

$$
f(\mathbf{r})=\left(\frac{a_{0}}{r}\right)^{2} \cos (2 \varphi)
$$

and the amplitude

$$
\beta_{1}=\frac{c a_{0}^{2}}{6} .
$$

This approximate expression is valid in the range $c r \ll 1$, i.e., at distances shorter than the curvature radius $1 / c$. We remark that at the contact line, the unperturbed profile $w_{0}$ and the deformation $\hat{\xi}$ differ by a factor 3 and thus are of the same order of magnitude.

The last term in Eq. (17) is readily integrated, and one finds for the area reduction due to the deformation field,

$$
-\frac{1}{2} \int_{0}^{2 \pi} d \varphi \hat{\xi} w_{0}=-\frac{\pi}{4} \beta_{1} c a_{0}^{2} .
$$

resulting in the total change of area

$$
S_{1}=-\pi a_{0}^{2}\left(1+\frac{c^{2} a_{0}^{2}}{8}+\frac{\beta_{1} c}{4}\right) .
$$

A colloidal particle at a flat interface reduces the area by $\pi a_{0}^{2}$; at a curved interface described by $w_{0}$ augments this quantity by a fraction $\frac{1}{8} c^{2} a_{0}^{2}$, whereas the interface deformation $\xi$ enhances this effect by $\frac{1}{4} \beta_{1} c$. Inserting the above relation for $\beta_{1}$ we obtain the total change of area,

$$
S_{1}=-\pi a_{0}^{2}\left(1+\frac{1}{6} c^{2} a_{0}^{2}\right) .
$$

The curvature-induced enhancement is proportional to the square of the aspect ratio $c a_{0}$ of the contact line and curvature radii.

The reduction of the undeformed interface area by a factor $\frac{1}{8} c^{2} a_{0}^{2}$ may be understood by noting that the area inside of a circle is always smaller than the area inside of a three-dimensional curve, obtained by distorting that circle on the sphere while keeping its center fixed. The second term in $S_{1}$, of relative magnitude $\frac{1}{4} \beta_{1} c$, arises from the relaxation of the liquid phase boundary and thus has no such simple explanation. Our calculation shows that both contributions diminish the interface area and are of the same order of magnitude.

\section{TWO-PARTICLE CASE}

Now we turn to the case where a second particle is found at the position $\boldsymbol{\rho}=\rho\left(\cos \varphi_{0}, \sin \varphi_{0}\right)^{\dagger}$. We evaluate the induced pair interaction for the case where the 
distance $\rho$ is larger than the contact line radius $a_{0}$ but smaller than the curvature radius,

$$
a_{0} \ll \rho \ll 1 / c .
$$

The first inequality justifies to retain only the term of leading order in $a_{0} / \rho$, whereas the second one implies that the principal curvatures at the positions of the two particles differ very little and may be taken to be identical.

\section{A. Deformation field}

If the prinicpal axes don't change over the distance $\rho$, the deformation fields of the particles are described by the same function $f$, and the solution of $\nabla^{2} \xi=0$ is a superposition

$$
\hat{\xi}(\mathbf{r})=\beta_{2}[f(\mathbf{r})+f(\mathbf{r}-\boldsymbol{\rho})] .
$$

The contribution of the particle at $\mathbf{r}=0$ reads

$$
f(\mathbf{r})=\left(\frac{a}{r}\right)^{2} \cos (2 \varphi-\delta)
$$

where the phase $\delta$ accounts for the coupling of the deformation field to the orientational angle $\varphi_{0}$. The field $f(\mathbf{r}-\boldsymbol{\rho})$ is due to the particle at position $\boldsymbol{\rho}$. Since we are interested in its values close to the origin $r \ll \rho$, we expand in powers of $r / \rho$ and truncate at quadratic order. This is done most simply after writing the field in cartesian coordinates, $f(\mathbf{R})=a^{2}\left(X^{2}-Y^{2}\right) /\left(X^{2}+Y^{2}\right)^{2}$, and we find

$$
f(\mathbf{r}-\boldsymbol{\rho})=f_{0}+\frac{3 a^{2}}{\rho^{4}} r^{2} \cos \left(2 \varphi-4 \varphi_{0}+2 \delta\right),
$$

where $f_{0}$ contains a constant and a term proportional to $\left(r a^{2} / \rho^{3}\right) \cos \left(\varphi-3 \varphi_{0}\right)$. The constant would merely shift the origin of the vertical axis, whereas the remaining term vanishes when inserting $(23)$ in (17); thus we discard $f_{0}$.

\section{B. Superposition approximation}

A widely used approximation consists in taking $\hat{\xi}(\mathbf{r})$ as the sum of two single-particle deformations, i.e., putting $\beta_{2}=\beta_{1}$ and $\delta=0$ in the above expression. Integrating the overlap term along the contact line $r=a_{0}$,

$$
\frac{\beta_{1}}{2} \int_{0}^{2 \pi} d \varphi f(\mathbf{r}-\boldsymbol{\rho}) w_{0}(\mathbf{r})=\frac{3}{4} \pi \beta_{1} c a^{2} \frac{a_{0}^{4}}{\rho^{4}} \cos \left(4 \varphi_{0}\right),
$$

the interface area (17) is readily evaluated and reads

$$
S_{2}=2 S_{1}-\frac{9}{2} \pi \beta_{1}^{2} \frac{a_{0}^{4}}{\rho^{4}} \cos \left(4 \varphi_{0}\right) .
$$

In terms of the interface energy $E=\gamma S$, this expression constitutes a pair interaction that depends both on the distance $\rho$ and the orientation $\varphi_{0}$.

\section{Boundary condition}

The superposition approximation is justified if the deformation field arises from an external force acting on the particles. In the present case, however, the deformation originates from the boundary condition imposed in terms of the contact angle. As a consequence, the prefactor $\beta$ is not the same for one and two particles. Here we solve the boundary conditions for the total deformation field of two particles that is given by (23-24).

In order to determine $\beta_{2}$ and $\delta$, we insert $\hat{\xi}(\mathbf{r})$ in Eq. (16) and evaluate $\hat{\xi}_{r}$ at the contact line of the particle at the origin, i.e., at $r=a_{0}$. The first term in (23) gives

$$
\left(a_{0} \partial_{r}-1\right) f(\mathbf{r})=-3 \cos (2 \varphi-\delta),
$$

whereas the second one leads to

$$
\left(a_{0} \partial_{r}-1\right) f(\mathbf{r}-\boldsymbol{\rho})=3\left(a_{0} / \rho\right)^{4} \cos \left(2 \varphi-4 \varphi_{0}+2 \delta\right) .
$$

After decomposing the cosine and inserting $w_{0}(\mathbf{r})$, we obtain for Eq. (16) the form $\cos (2 \varphi) Q-\sin (2 \varphi) P=0$ which is valid for all $\varphi$. The resulting equation $Q=0$ reads explicitly

$$
\frac{1}{2} c a_{0}^{2}-3 \beta_{2}\left(\cos \delta-\frac{a_{0}^{4}}{\rho^{4}} \cos \left(4 \varphi_{0}-2 \delta\right)\right)=0,
$$

whereas $P=0$ gives

$$
\sin \delta-\left(a_{0} / \rho\right)^{4} \sin \left(4 \varphi_{0}-2 \delta\right)=0 .
$$

To leading order in the small parameter $a_{0} / \rho$, one finds the phase $\delta=\left(a_{0} / \rho\right)^{4} \sin \left(4 \varphi_{0}\right)$. Inserting this phase in (23) and integrating (17), one finds corrections of the order $\delta^{2} \sim\left(a_{0} / \rho\right)^{8}$ which are negligible at distances $\rho$ larger than the particle size.

Putting $\delta=0$ in the relation for $\beta_{2}$ one finds the prefactor of the deformation field

$$
\beta_{2}=\frac{c a_{0}^{2}}{6} \frac{1}{1-\psi}=\frac{\beta_{1}}{1-\psi}
$$

with the shorthand notation

$$
\psi=\left(a_{0} / \rho\right)^{4} \cos \left(4 \varphi_{0}\right) .
$$

The quantity $\beta_{2}$ is maximum for particles aligned on one of the principal curvature axes. In the limit $a_{0} / \rho \rightarrow 0$, we recover the deformation amplitude (22) of a single particle.

\section{Area reduction}

Inserting (23) and (26) in Eq. (17), one readily obtains the change of area in the presence of two particles

$$
S_{2}=-2 \pi a_{0}^{2}\left(1+\frac{c^{2} a_{0}^{2}}{8}+\frac{\beta_{2} c}{4}\right) .
$$


Evaluating the corrections with respect to the singleparticle expression $S_{1}$, we find in leading order in the small parameter $\psi$

$$
S_{2}-2 S_{1}=-\frac{\pi}{2} \beta_{1} c a_{0}^{2} \psi=-3 \pi \beta_{1}^{2} \psi
$$

This is by a factor $\frac{2}{3}$ smaller than the result (25) from the superposition approximation.

These results are readily generalized to the case of $N$ particles. Solving the boundary condition for the superposition of $N$ one-particle deformation fields, we obtain the change of interface area

$$
S_{N}=N S_{1}-3 \pi \beta_{1}^{2} \sum_{<i, j>} \psi_{i j} .
$$

Here the correction factor $\psi_{i j}$ depends on the distance and relative orientation of particles $i, j$, and the sum runs over all pairs.

\section{DISCUSSION}

The presence of the colloidal particles reduces the area of the liquid interface by $S$ and thus diminishes the energy by the amount

$$
E=\gamma S
$$

As discussed below Eq. (1) this expression does not depend explicitly on the particle-fluid interface areas $P_{1}$ and $P_{2}$, since their effect has been absorbed in the constraint of Young's law. More precisely we have discarded a constant term $\frac{1}{2}\left(\gamma_{1}+\gamma_{2}\right)\left(P_{1}+P_{2}\right)=\left(\gamma_{1}+\gamma_{2}\right) 2 \pi r_{0}^{2}$, which does not depend on the contact line and thus is irrelevant for our purpose.

We first discuss the energy gain due to a single particle, and then consider the deformation-induced effective pair interaction of nearby particles.

\section{A. Single-particle energy}

From the single-particle area (22) one readily obtains the energy gain

$$
E_{1}=\gamma S_{1}=-\pi \gamma a_{0}^{2}\left(1+\frac{c^{2} a_{0}^{2}}{6}\right) .
$$

The leading term of the trapping energy reads $E_{0}=$ $-\pi \gamma a_{0}^{2}$. For a micron size particle $\left(a_{0}=1 \mu \mathrm{m}\right)$ at an interface with a tension $\gamma=100 \mathrm{~mJ} / \mathrm{m}^{2}$, it is of the order of $E_{0} \sim 10^{-13} \mathrm{~J}$, which is about $10^{8}$ times the thermal energy $k_{B} T$ at room temperature.

Here we are mainly interested in the contribution that depends on curvature,

$$
E_{1}-E_{0}=-\frac{\pi}{6} \gamma c^{2} a_{0}^{4}
$$

At an interface with curvature radius $1 / c \sim 100 \mu \mathrm{m}$, this energy difference takes a value of about $4 \times 10^{-18}$ $\mathrm{J}$, which is by three orders of magnitude larger than the thermal energy at room temperature, $E_{1}-E_{0} \sim 10^{3} k_{B} T$. Two conclusions may be drawn from this estimate. First, in the case of a non-uniform curvature $c(\mathbf{r})$, the energy $E_{1}-E_{0}$ varies with position and constitutes an effective potential that takes its minimum value where the curvature is maximum; this potential provides a lateral force,

$$
F(\mathbf{r})=-\nabla E_{1}(\mathbf{r})=\frac{\pi}{3} \gamma a_{0}^{4} c \nabla c,
$$

that pushes the floating particle towards regions of maximum curvature. With the above parameter values one finds $F \sim 10^{-14} \mathrm{~N}$. Second, the deformation energy, i.e., the term arising from the profile $\hat{\xi}$, is of the order of the curvature-dependent term, $E_{1}-E_{0}$, and thus exceeds by far the thermal energy; we conclude that the deformation field $\hat{\xi}(\mathbf{r})$ is hardly affected by thermal fluctuations.

Regarding the validity of the small-gradient approximation, leading-order corrections are of the order $\mathcal{O}\left(\left(c a_{0}\right)^{4}\right)$ and thus by a factor $c^{2} a_{0}^{2} \sim 10^{-4}$ smaller than the above results.

\section{B. Pair interaction}

Two nearby particles interact through the interface induced potential energy $U=\gamma\left(S_{2}-2 S_{1}\right)$. With (27) one finds the expression

$$
U\left(\rho, \varphi_{0}\right)=-\frac{\pi}{12} \gamma \frac{c^{2} a_{0}^{8}}{\rho^{4}} \cos \left(4 \varphi_{0}\right),
$$

that depends both on the distance $\rho$ and the relative orientation $\varphi_{0}$ with respect to the principal curvature axes. Formally, the expansion in powers of $a_{0} / \rho$ breaks down if the two particles are at contact, $\rho=2 r_{0}=2 a_{0} / \sin \theta_{0}$. Even in this extreme case, the above expression for $U$ provides a meaningful estimate for the interaction energy. Yet in real systems, the particles are often subject to short-range repulsive forces, arising from surfactants grafted at the surface or from the screened electrostatic of their charges, that keep them at a minimum distance of a few radii, where our result applies. With the parameter values given above and at distances of a few particle radii, the interaction potential is of the order of $10^{-19} \mathrm{~J}$ which is still larger than the thermal energy.

Thus the interaction energy may induce aggregation and ordering of the particles. Since $U$ is minimum for $\varphi_{0}=0$ and $\frac{\pi}{2}$, its angular dependence favors alignment parallel to the principal axes. In the case of several particles, one expects the formation of colloidal clusters of cubic symmetry, as shown schematically in Fig. 4.

\section{Catenoid}

The present work applies to colloidal particle suspended at an arbitrary surface of zero mean curvature. 


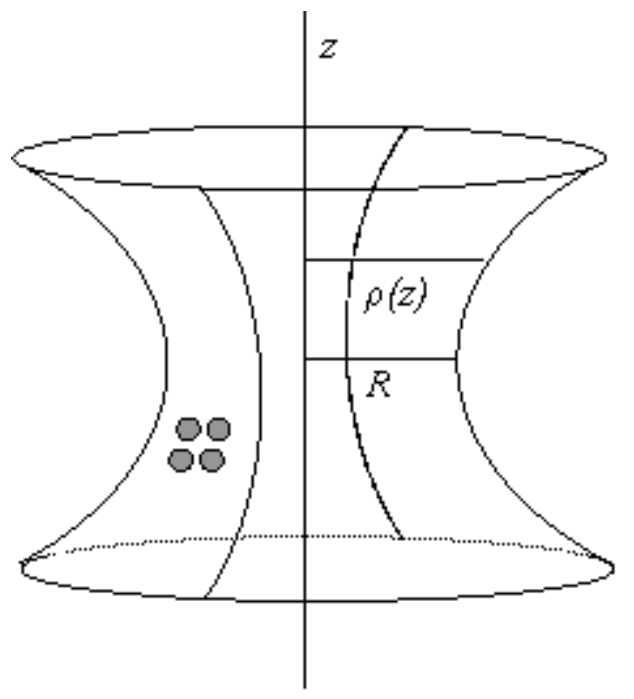

FIG. 4: Catenoid, or surface of revolution of a catenary, with the parameterization $\rho(z)=R \cosh (z / R)$. As explained in the text, capillary forces may result in colloidal aggregates of cubic symmetry.

Here we discuss several particular features that arise for a catenoid, i.e., for the surface of revolution of the catenary about the $z$-axis. A simple parameterization is given in terms of distance from the vertical axis,

$$
\rho(z)=R \cosh (z / R),
$$

as shown in Fig. 4. The minimum radius $R$ occurs at midplane $z=0$. The principal curvatures are of opposite sign, $c_{u}=c=-c_{v}$, and depend on the vertical position $z$ as

$$
c=\frac{1}{R \cosh (z / R)^{2}},
$$

and so does the trapping energy $E_{1}$. From (28) it is clear that the energy $E_{1}$ takes its minimum value at $z=0$ where the curvature is largest. The vertical force $F=$ $-d E_{1} / d z$

$$
F(z)=-\frac{4 \pi}{3} \frac{\gamma c^{2} a_{0}^{4}}{R} \tanh (z / R)
$$

pushes the particles towards midplane where the curvature takes its maximum value $c=1 / R$.

For micron-sized particles, the curvature-induced force is comparable to gravity. If the maximum value of $F(z)$ exceeds $m g$, the height-dependent potential energy $E_{1}(z)-E_{0}+m g z$ of a buoyant particle of effective mass $m$ presents a metastable minimum; its position $z_{0}$ is determined by putting $F\left(z_{0}\right)=m g$. Thus one expects that colloidal particles on a vertically placed catenoid accumulate at a height $z_{0}<0$ below midplane. This is illustrated in Fig. 5 for particles of radius $a_{0}=1 \mu \mathrm{m}$ at a catenoid of minimum radius $R=100 \mu \mathrm{m}$. The total

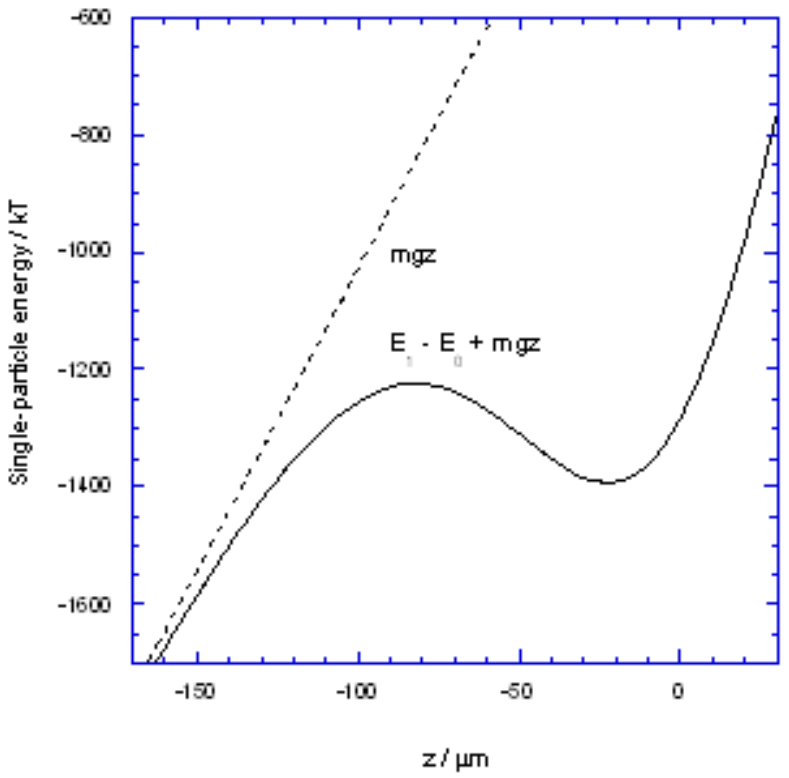

FIG. 5: Total potential energy $E_{1}-E_{0}+m g z$ of a colloidal particle of radius $r_{0}=1 \mu \mathrm{m}$ and density $\varrho=1 \mathrm{~g} / \mathrm{cm}^{3}$ trapped at a vertical catenoid, with a contact line radius $a_{0}=1 \mu \mathrm{m}$. The interface parameters are $\gamma=100 \mathrm{~mJ} / \mathrm{m}^{2}$ and $R=100$ $\mu \mathrm{m}$. There is a local minumum at $z_{0} \approx-25 \mu \mathrm{m}$, which is stabilized by a barrier of about $170 k_{B} T$. The dashed line indicates the gravitational potential $m g z$.

energy is shifted towards negative values by the curvature contribution $E_{1}(z)-E_{0}$, with respect to the gravitational potential $m g z$. The metastable minimum occurs at $z_{0} \approx-25 \mu \mathrm{m}$ and is stabilized by a barrier of about $170 k_{B} T$.

\section{Finite mean curvature}

We close with a brief discussion of interfaces with finite mean curvature; as the simplest realization we take the case where one of the principal curvatures vanishes, $c_{u}=c$ and $c_{v}=0$. Then the Laplace equation (15) is replaced with the inhomogeneous relation $\nabla^{2} \xi+c=0$, which just expresses the fact the cylinder is not a minimal surface. Yet such interfaces do occur as a metastable state, for example in liquid jets, or in the presence of external forces, such as hydrodynamic coupling of twophase microfluidic devices or gravity at the meniscus of a liquid surface. Two aspects of the present work could be of some relevance for these systems.

Liquid jets are inherently unstable and decay into droplets due to the tension-driven Rayleigh instability; its onset is triggered by tiny surface fluctuations. The presence of a colloidal particle leads to a local deformation that could develop much quicker than the fluctuation-induced perturbation and thus could significantly accelerate the instabililty. 
The viscous flow of two different fluid phases in a microchannel may result in a stable cylindrical interface. Then the Laplace equation is supplemented with a restoring force, $\nabla^{2} \xi+c+R[\xi]=0$, that arises from hydrodynamic constraints. Still, the presence of the colloidal particles would deform the interface and lead to a local depression $\hat{\xi}(\mathbf{r})$ of the interface profile $\hat{\xi}(\mathbf{r})$.

Finally we mention a striking example of a curvatureinduced lateral force, which has been shown to operate in the meniscus climbing skills of water-treading insects and amphiphile beetle larvae $[5,6]$. In order to pass from the water surface to land and to overcome the slippery meniscus, the larvae deform their body in such a way that the contact line becomes a 3D curve that fits in the upper part of the concave meniscus. Then the interface deformation energy is smallest where the curvature is largest, and thus provides a lateral force pushing the larva uphill towards the shore. The above expression for a catenoid shows that the tension-induced force $F \sim c^{2} r_{0}^{4}$ varies more strongly with the size of the floating object than gravity $m g \sim r_{0}^{3}$; in spite of the somewhat smaller curvature $c$, the effects on millimeter sized animals exceed those discussed above for colloidal particles.

\section{SUMMARY}

We have studied how the presence of spherical particles affects a liquid interface with two different principal curvatures $\pm c$. In the absence of external forces, the curvature of the liquid phase boundary leads to several novel capillary phenomena.

(i) The contact line of a particle at a curved interface $w_{0}$ would not satisfy the condition of a constant contact angle. Mechanical equilibrium thus requires a finite deformation which, in turn, reduces the interface energy.

(ii) The single-particle energy (28) varies with the square of the curvature and takes its mimimum value where the curvature is largest. Thus there is an effective force pushing the particles towards strongly curved regions of the interface.

(iii) The superposition of the deformation of two nearby particles results in an anisotropic curvaturedriven interaction $U$. Its angle-dependence favors the formation of colloidal aggregates of cubic symmetry, whereas the capillary interactions of spherical particles studied so far are isotropic and result in hexagonal clusters.

(iv) For micron sized particles on a vertical catenoid interface, the competition of the curvature-driven force and gravity results in a rather deep metastable minimum of the total energy; one expects colloidal particles floating on the interface to be captured slightly below the midplane of the catenoid.

Stimulating discussions with Yacine Amarouchène are gratefully acknowledged.
[1] P. Pieranski, Phys. Rev Lett. 45, 569 (1980)

[2] B.W. Russel, D.A. Saville, W.R. Schowalter, Colloidal Dispersions (Cambridge University Press 1989)

[3] F. Burmeister et al., Appl. Surf. Sci. 144-145, 461 (1999)

[4] C. Zeng, H. Bissig, A.D. Dinsmore, Solid State Comm., in press (2006)

[5] D.L. Hu, J.W.M. Bush, Nature 437, 733 (2005)

[6] J.W.M. Bush, D.L. Hu, Ann. Rev. Fluid Mech. 38, 339 (2006)

[7] R. Aveyard, et al., Phys. Rev. Lett. 88, 246102 (2002)

[8] M.G. Nikolaides et al., Nature 420, 299 (2002)

[9] M. Megens, J. Aizenberg, Nature 424, 1014 (2003)

[10] L. Foret, A. Würger, Phys. Rev. Lett. 92, 058302 (2004)

[11] M. Oettel, A. Dominguez, S. Dietrich, Phys. Rev E 71,
$051401(2005)$

[12] K.D. Danov, P.A. Kralchevsky, M.P. Boneva, Langmuir 22, 2653 (2006)

[13] A. Würger, to appear in Europhys. Lett. (2006)

[14] P.A. Kralchevsky, K. Nagayama, Adv. Coll. Interf. Sci. 85, 145 (2000)

[15] D. Stamou, D. Duschl, D. Johannsmann, Phys. Rev. E 62, $5263(2000)$

[16] J.-B. Fournier, Phys. Rev. E 65, 031601 (2002)

[17] E.A. van Nierop, M.A. Stejnman, S. Hilgenfeldt, Euorphys. Lett. 72, 671 (2005)

[18] J.C. Loudet, A.G. Yodh, B. Pouligny, Phys. Rev. Lett. 97, 018304 (2006)

[19] J.C. Loudet et al., Phys. Rev. Lett. 94, 018301 (2005) 